\title{
The Instructional Use of Computers in Dental Education
}

\author{
WILLIAM F. FITZGERALD \\ CAIDENT Project, Department of Educational Resources, School of Dentistry, Uni- \\ versity of Michigan, Ann Arbor, Michigan 48104, U.S.A.
}

(Received 3 May 1973)

\begin{abstract}
The formation, development, and current status of a major instructional materials preparation project, the CAIDENT Project, at the School of Dentistry, The University of Michigan, is discussed. A rationale for the place of individualized instruction in general and CAI in particular is presented. Topics include staffing strategies and ways to encourage faculty participation. Examples are included to illustrate operational CAI programs at Michigan in Pedodontics and Oral Diagnosis. Comments about the future of CAI in the Health Sciences are included.
\end{abstract}

Computer-assisted instruction Faculty involvement Staffing
CAIDENT project Pedodontics Oral diagnosis Computers in dentistry Individualized instruction

Dentistry

\section{THE CAIDENT PROJECT}

\section{History}

In 1970, the School of Dentistry at the University of Michigan acquired an IBM 1130 computer for patient records management, to keep track of student progress, and to match patients' needs with students' requirements. Later, a small multiplexor (Western Telematics) with two lines was connected, two low-cost CRT (television display) terminals were bought and a programmer was hired. Because the computer was purchased for administrative purposes exclusively, early CAI experimentation was conducted during off-hours and evenings. The Oral Cancer recognition program was adapted from Ohio State University, faculty were invited to see this new teaching medium, and CAI was officially introduced to our school. Evaluation of the operating characteristics of this system indicated that: (1) it didn't switch control from terminal to terminal fast enough; (2) it was limited in size (16 terminals, maximum); (3) there wasn't enough storage; and (4) we would not be allowed to use the computer except noons and evenings. For these reasons, use of this system was limited to demonstration and a search for operational computer access was initiated.

\section{Development}

Phase II began in the Fall of 1971 when CAI was included as part of a larger movement: the Pilot Program in Dentistry (PPD). PPD was designed locally to allow a random sample of students to study at their own pace and to proceed in the curriculum based on successful completion of tests and other performance measures. A position paper on how to design, devclop and deliver individualized instructional materials to dental students, an "operating manual for PPD”, was developed. Some of the aspects of the problem were quantitatively 
estimated and mathematically massaged.* Various documents (the position paper, the quantitative estimates, projections and a few others) were organized into a coherent whole as a grant request to the Bureau of Health Manpower Training. CAI was originally estimated at 30 per cent of the total delivery of instructional materials. Work to date, March 1973 , suggests that the final mix of media will include probably only about 20 per cent CAI. To put this percentage in perspective, a small portion of the original analysis of projected media delivery is presented below.

Michigan's Dental curriculum now contains in excess of 4000 (clock) hr of scheduled time for students. Of that time, 1520 are essentially lecture hours. If 50 per cent of the lecture hours were amenable to communication via teacher-absent instructional materials, it would mean the development of $760 \mathrm{hr}$ (lecture-hour-equivalent time) of instructional products. Of all the media available, videotape, slides, audio-tutorial instruction, etc., it is estimated that CAI will be the delivery medium of 30 per cent of those hours. This can be interpreted to mean that the average student, between his arrival to and his graduation from our dental school, can be expected to spend $258 \mathrm{hr}$ interacting with a computer for instructional purposes.

Although our current estimates have reduced that projection somewhat, it is still quite likely that our students will spend much time at a computer terminal because of increased emphasis on diagnostic testing and instructional strategy management. These two uses of a computer, testing and management, are now seen as increasing in importance because of the emphasis on student-directed learning. Our expectations for this future environment include student-initiated testing on diagnostic computer programs that will advise a student of his strengths and weakncsses and suggest resources for learning. The student himself then may elect any of a number of strategies to achieve his mastery of the curriculum. At various checkpoints he will interrogate a cumulative record of his performance and this management program will advise him of deadlines by which various requirements must be completed if he wants to maintain a certain degree accomplishment pace for himself. While these computer uses are not the focal point of this paper, they are mentioned because they will require computer access which must be anticipated.

\section{Current status}

The Special Improvement Grant request was approved, and funding was received on 8 August 1972. The grant provides for a stand-alone computer (Digital Equipment Corporation PDP-10) or equivalent computer access, 32 terminals, communications lines and staff. At this time, final analyses are being conducted to determine the cost/effectiveness of acquiring a stand-alone computer to be housed and operated by the dental school, compared with cost-sharing achieved by joining other projects and sources of financial support to provide campus-wide centralized CAI service. There is an emphasis on the latter alternative but the matter is still under investigation.

The proposal for this grant identified a 5-yr implementation scheme, with the addition of 6-8 computer terminals each year. The project now has seven terminals and a full-time applications programmer. Three more applications programmers and a full-time systems programmer will be added shortly and by September of 1973 the terminal capacity will be

\footnotetext{
*Reported at the Summer, 1972 Convention of the Association for the Development of Instructional Systems (ADIS); now the Association for the Development of Computer-based Instructional Systems (ADCIS); under the mis-title of "Cost/benefit Analysis of CAI in a Dental School". Copies of that paper and of its successor are available on request from the author.
} 
close to 16 in number. In addition, a learning laboratory (the CAIDENT Center) is under construction, and this facility will house the terminals as well as slide projectors, videotape cassette players and other audio-visual equipment.

\section{Staffing}

The personnel required for a medium-size operation such as this fall basically into two categories: operations and programming. The operations staff size and constituency is highly dependent upon the type and features of the particular computer selected, and, because of this variability, will not be discussed here. The programming staff requirements are determined by two basic factors: (1) the mix of tasks subsumed by each person; and (2) the extent of faculty or other content expert involvement.

For the sake of argument consider two extreme models: "generalist" and "specialist". In the generalist model, a "programmer" would attend lectures and take tests, duplicating the experience of a student. From those experiences he would organize the content into colateral and hierarchical (linear) sequences, identifying what material should be taught in sequence and what could be taught before or after other content. This process, instructional programming, can become extensive and results in a logical information flow with decision or "branching" points. Once established, this logical structure must be coded into a computer language, so our generalist now assumes the role of computer programmer and generates a computer source language version of the same content expressed in logic.

The specialist approach divides the functions into job specialties. Such personnel are identified as (1) content expert (faculty), (2) instructional programmer and (3) computer programmer. It is this specialist model which seems to have the greatest cost/effective payoff in large-scale production environments; in the CAIDENT Project we are now setting up the data collection system that will enable us to precisely calculate the cost/effectiveness of this approach.

\section{Faculty involvement}

There are many ways in which faculty can be motivated (in the broadest sense) to serve as content experts. These will be presented with comments on their value and effectiveness.

(1) Walk-in. This is usually the best if it results from personal interest or initiative. In this classification a faculty member finds that some educational problems do not seem to respond to his efforts. This motivates him to contact one of the senior instructional development staff who assists in analyzing the problem and suggesting solutions. If CAI is part of the solution, all the better. Although it does have occasional problems ("I want to put something on computer" [sic]) it is frequently the reflection of some familiarity with the advantages of CAI and a suspicion that CAI may be an appropriate solution. Incidently, it is frequently advantageous to accommodate tangential requests such as making slides or graphs on the understanding that future participation in developing instructional (CAI) materials will be expected.

(2) Pyramid subscription. This technique is concisely expressed in the words of an instructional programmer as spoken to a faculty member: "Don't thank me; just advertise what you've done and get two of your colleagues to help us develop materials." If adroitly but sincerly handled, this can be very effective.

(3) Executive mandate. These come from department chairmen, heads of curriculum committees, and deans and have mixed results. Since there is often some degree of suspicion, defensiveness or resentment, initial communications are peppered with explanations and 
demonstrations. In general, there are more subtle but more effective ways to express the same message.

(4) Competition. There is the occasional faculty member whose self-image contains such descriptors as innovative, flexible, experimental, leader, etc. While an overture from a person like this may not be based on the "right" reasons, there is, nonetheless, eagerness which should not perish. Above all we have found that faculty interest is a perishable commodity.

(5) Promotion incentive. The vast majority of professorial faculty are promoted and otherwise recognized on the basis of research and publications. It is difficult, if not impossible, to satisfactorily answer the question "Why should I spend time developing an instructional package when that will reduce my research/writing/publishing time and hence potentially slow down my promotions?" Even if appropriate recognition is provided within a single institution, there still remains the problem of positions in other schools if transfer is considered. Clearly this will neither be solved overnight nor receive universal acceptance. And it certainly won't happen until some faculty add a section to their vita titled "Instructional Programs Produced" and describe these efforts proudly to reflect the extensive time and energy spent.

\section{CAI IN DENTISTRY}

\section{The need}

Dentistry, like other professions, is both an art and a science in which very high standards of performance and excellence are required and expected. Surely no one would be eager to go to a dentist who was a " $\mathrm{C}$ " student; graduation, certification and state licensure require and assure that each practitioner meets or exceeds exacting standards in all areas of dental performance. And yet herein lies the problem since it is a well documented fact that 1 st-yr dental students vary substantially in their respective abilities to perform the skills endemic to the profession. This specific point-that people are different-justifies individualized instruction in general and CAI in particular, for how else can variable inputs (differing student abilities) proceed through a uniformly applied process (lockstep curriculum) and result in a constant output (all students surpassing uniformly high standards)? The answer is that they can't. Analytically, this is undeniable. The application of this simple model to current dental instruction requires the explanations that: (1) dental school is not a constant process; some students study harder, receive more attention and spend more time than others; and (2) the output is neither constant nor variable but in direct proportion to the input; while there are differences in abilities among students at graduation, students are at least more homogeneous after than before this process. The point of all this is that while attempts are being made to make the process variable and adaptive to students, sometimes (and for many reasons) the right instruments to maximize that flexibility to respond to these variables are not being used. And here is where CAI comes in. But before the role of CAI can be extolled, two other factors must be examined: how do students learn, and for what learning material is CAI appropriate?

Learning can be conceptualized as an output based on a number of inputs: (1) time required to learn (learner intelligence); (2) time provided for learning (both information reception and "memory consolidation"); (3) student motivation; (4) quality of information presentation; and (5) environmental factors (noise, light, heat, hunger, distraction, anxiety, 
etc.). Each of these factors could be discussed at great length but they are presented here only to focus on point (4) in contrast to the others.

Regrettably media has seen much attention in preference to better (educational) problem analysis. This author has had the embarrassing experience of having a faculty member ask for "something on computer" to discover an infatuation with hardware prior to a determination of the problem and a sound basis for identifying some computer method as the vehicle to implement the solution. If you need to teach location of instruments on the operatory tray, you can probably succeed best with slides or some other still visual. To show wrist action in sharpening instruments you'll succeed best (most quickly arrive at measured student learning performance) with some form of moving visual-inefficiently with live demonstration; efficiently with videotape or movie film. So what is the problem for which CAI is the solution?

\section{Uses of CAI in dentistry}

Computer Assisted Instruction (CAI) is particularly appropriate where: (1) students require substantially different amounts of time to learn; (2) different responses are made by students to the same question; (3) students need to explore the effects of various decision strategies; (4) actions taken on the basis of student response can be identified; (5) each student is required to actively participate in the specification of or commitment to a particular decision; (6) it is cumbersome, difficult or unnecessary to present all cues which comprise a particular problem situation; and (7) material is substantially repetitive. While there are other "border" conditions which must be met, these foregoing seven factors influence about 90 per cent of the decision to use CAI as a solution to certain problems. The role of these factors in various decisions will be explained through examples:

\section{Example I}

Problem. Approximately every 3 weeks, 15 junior students rotate into Pedodontics clinic. At that time they receive special training in all aspects of dental practice with children, including the analysis of a child's diet to identify foods that are high in refined sucrose which contributes to the formation of caries. Students receive a lecture on this topic and a few examples of dietary analysis are presented. Students are required to perform this skill in the clinic.

One solution. Provide each student with a sample diet, require that he conduct the analysis on paper and submit this to an instructor for examination and criticism (redirecting feedback).

The CAI Solution. A computer program was written which asked the student to enter each component step in the analysis for immediate evaluation. The presumption in this case is that a student can reach the wrong conclusion in many ways and the best use of the student's time and the best way to block the student from habitually analyzing components incorrectly is to indicate his errors as he makes them. In this case, students are provided with a set of seven diet histories, a table of sucrose contents of various foods and access to the computer. Armed with these resources, students are asked to:

(1) using a pencil, circle all the foods that contain sucrose (on the diet history)

(2) identify days in which the diet is representative (to the computer)

(3) indicate the high sucrose-containing foods on representative days (to the computer)

(4) indicate substitutes for foods identified in step 3 (in writing).

The cases require progressively finer discrimination skills, and each student is expected to 
Welcome to the CAIDENT Terminal System. ${ }^{1}$

(Student signs on ...)

You may choose any of the following programs:

1. Oral Histology (Dr. Avery and Dr. Straclian)

2. Histology

3. Organology

4. Utilization of Laboratory Studies (Dr. Gobetti)

5. Oral Diagnosis Senior (Dr. Millard)

6. Pedôdontics Dietary History Program

7. Pedodontic Radiographic Interpretation Program (Soph. Pedo 642)

8. Oral Surgery Program.

Type the number of the program you select and press the "CR"

S: 6

(. . selects Pedodontics program)

One moment please while I get your program.

$\dagger+3$

Welcome; this is the Pedodontics Dietary History and Review ${ }^{4}$ Program.

If this is the first time you have used the terminal, read the manual to

familiarize yourself with it.

Press the "CR" key when you are ready.

Is this the first time you have worked on this program?

S: Yes

t†

We shall start with Case 1 .

CASE 1

Mrs. Yazzie and her child Paul came in for an initial interview a week

ago. At that time you showed her how to fill out a dietary history form.

It is now one week later, Mrs. Yazzie has handed you the form. Your

task now is to prepare a review of the diet for her. From the dietary

forms given to you, turn to the dietary history form for Paul Yazzie.

Circle all refined sucrose containing foods in this dietary history.

Please Note: Any dietary history may reveal foods that contain natural sucrose. This program is designed to identify foods containing refined sugar (sucrose). (Press the "CR" key after you have circled all items.) $+\dagger$

From the dietary history, what days should not be used because of unusual circumstances. (Type the number of a day, (i.e. 1 or 2 or 3...), or type "none" and press the "CR" key.)

S: 2

You are right, 2 is an unusual day. Are there any more unusual days?

(Type another day or none.)

S: 4

Your are right, 4 is an unusual day. Are there any more unusual days?

(Type another day or none.)

S: None

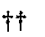

Very good, you now know all the unusual days in this dietary history.

On the basis of this information would you consider this an adequate

dietary history for this patient and her family? (Type yes or no)

s: Yes

Correct. There are at least three typical days in this dietary history. You should continue with your review with Mrs. Yazzie as soon as possible. (Press the "CR" key to continue) t广

Now evaluate the typical days in this diet history. Please turn to day 3 and examine the foods you have circled. What foods for this day contain refined sucrose? (Please refer to the foods by number and not by name) Enter the numbers one at a time

S: 14

Right, 14 is a sucrose containing food. Are there any more refined sucrose containing foods for this day? (Type another food number or type "none")

(current information on the CRT screen is refreshed until student indicates that he is ready to proceed)

(Student has been provided with a filled-out dietary history form off. line)

(Day "2" included a Halloween party and is not representative of this patient's diet)

(Patient did not eat breakfast or lunch on this day)

(The criterion is at least three representative days among five)

${ }^{1}$ This access system and the following program were programmed in FORTRAN by Judson Spencer.

${ }^{2}$ The "CR" key is a single button on the keyboard of the Hazeltine 2000A used to deliver this program. "CR" refers to "Carriage Return".

${ }^{3}$ These symbols indicate that the CRT screen is erased of all information.

4This program was developed by Drs. Loos, Straffon and Hartsook of the Department of Pedodontics, School of

Dentistry, University of Michigan. The text is reprinted with the permission of these authors. 
S: 4

You are wrong, 4 does not contain refined sugar (Sucrose). Please try again. (Type

another food number or type "none")

S: 5

Right, 5 is a sucrose containing food. Are there any more refined sucrose containing

foods for this day? (Type another food number or type "none")

S: 9

Right, 9 is a sucrose containing food. Are there any more refined sucrose containing fouds for this day? (Type another food number or type "none")

S: None

There are more sucrose containing foods for this day. Please examine your dietary history again. (Type another food or type "none")

S: 13

You are wrong, 13 does not contain refined sugar (sucrose). Please try again. (Type another food number or type "none")

S: 3

Right, 3 is a sucrose containing food. Are there any more refined sucrose containing

foods for this day? (Type another food number or type "none")

S: None

Correct, you have identified all the refined sucrose containing foods for this day.

(Press the "CR" key to continue)

$\dagger \dagger$

Realizing that most parents and children will brush their teeth after a main meal, the areas of concern are "snack time." Therefore, if tooth brushing occurs regularly after the main meals, then the elimination or minimization of foods containing refined sucrose should be recommended at snack time. On day 5 , what foods would you recommend to be eliminated at snack times? (Enter food numbers one at a time)

S: 8

Right. What other foods would you recommend to be eliminated? (Type another number or "none")

S: None

Right, you have identified all the foods that should be eliminated from this diet at snack times. (Press the "CR" key to continue)

t†

On your dietary review form, fill in the foods you would reconmend be eliminated at snack times for the entire week. (Press the "CR" key to continue)

$+t^{+}$

On the basis of your examination and radiographs, is the decay rate consistant with the sucrose intake reported on the history? Answer yes or no

S: Yes

Right, the sucrose intake for this child is consistant with his reported DMF. (Press the "CR" key to continue)

$\dagger \dagger$

Other than snack time, is there an excessive intake or refined sucrose containing foods for this child? Answer yes or no.

S: Yes

Wrong, check the regular meals again for refined sucrose containing foods. Refer to your dietary review form for substitutes you could suggest to further reduce the sucrose intake for this child. Although some of these foods are not popular with most children, the list is comprehensive enough for most families. (Press the "CR" key to continue) t†

When presenting the dietary review to the parents, remember that diet control

is one part of a good preventive dental program, especially if the sugar intake

is considered excessive. There are many different sugars and chemical

sweetners. Read the labels on foods and food products. Sucrose, which

is table sugar, supports plaque formation and caries best. Avoid sucrose sweetened foods at snack times especially in the sticky or gummy form. Good oral hygiene is an obvious sequel to the proper planning of meals. If a severe dietary or nutrition situation is encountered, refer these problems to a pediatrician or nutritionist.

(Please press the "CR" key)

it

The U.S. National Health Statistics for non-institutionalized children between

the ages of 6 and 11 years reveal a mean caries attack rate of 1.4 for the permanent dentition and a mean caries at tack rate of 3.0 for the primary dentition. In the Department of Pedodontics, the parent or guardian of all new patients will be asked to report a 5 day dietary history and the students will be expected to review the histories of patients assigned to him. The Department recommends a dietary history and review for those children whose caries attack rate exceed the National Health Statistics. (Press the "CR" key to continue) (no. 4 is cider)

(no. 5 is cupcake)

(no. 9 is lemonade)

(no. 13 is German potato salad)

(no. 3 is doughnut)

(no. 8 is candy)

(Note: DMFT .. decayed, missing and filled teeth count is provided on the diet form)

(Since this is the first case, there is a substantial repetition of information which was presented in lecture. Succeeding cases do not repeat all this information) 
respond to the complete set. Since this is actually practicing a skill which has been taught in class, it is technically the lowest level of CAI, "drill and practice". Anecdotally, students report that the program trains them to handle this skill automatically so that in the clinic they can focus their attention on the interpersonal communication with the parent. A sample of the interaction in this program is presented below.

Discussion. This program sample illustrates a simple interaction in which a student is asked to demonstrate the rather (educationally) elementary skills of finding sucrose contents from a table, and determining the representativeness of days for diet analysis. A computer interaction is appropriate because students do differ in their rate of learning or performance of these skills; students are asked to make a commitment based on judgment in which their options are few; the material needed to teach/practice these skills is repetitive; and it is not necessary to present the entire setting (a "real live" mother, etc.) to elicit the requisite student performance.

\section{Example II}

Problem. Students receive lectures and case presentations on Oral Diagnosis, but much of this is group activity in which each student does not have the opportunity to express his opinion and get verification or explanation of why his diagnosis was correct or incorrect. Individual attention is available in the clinic setting but students are limited by the number

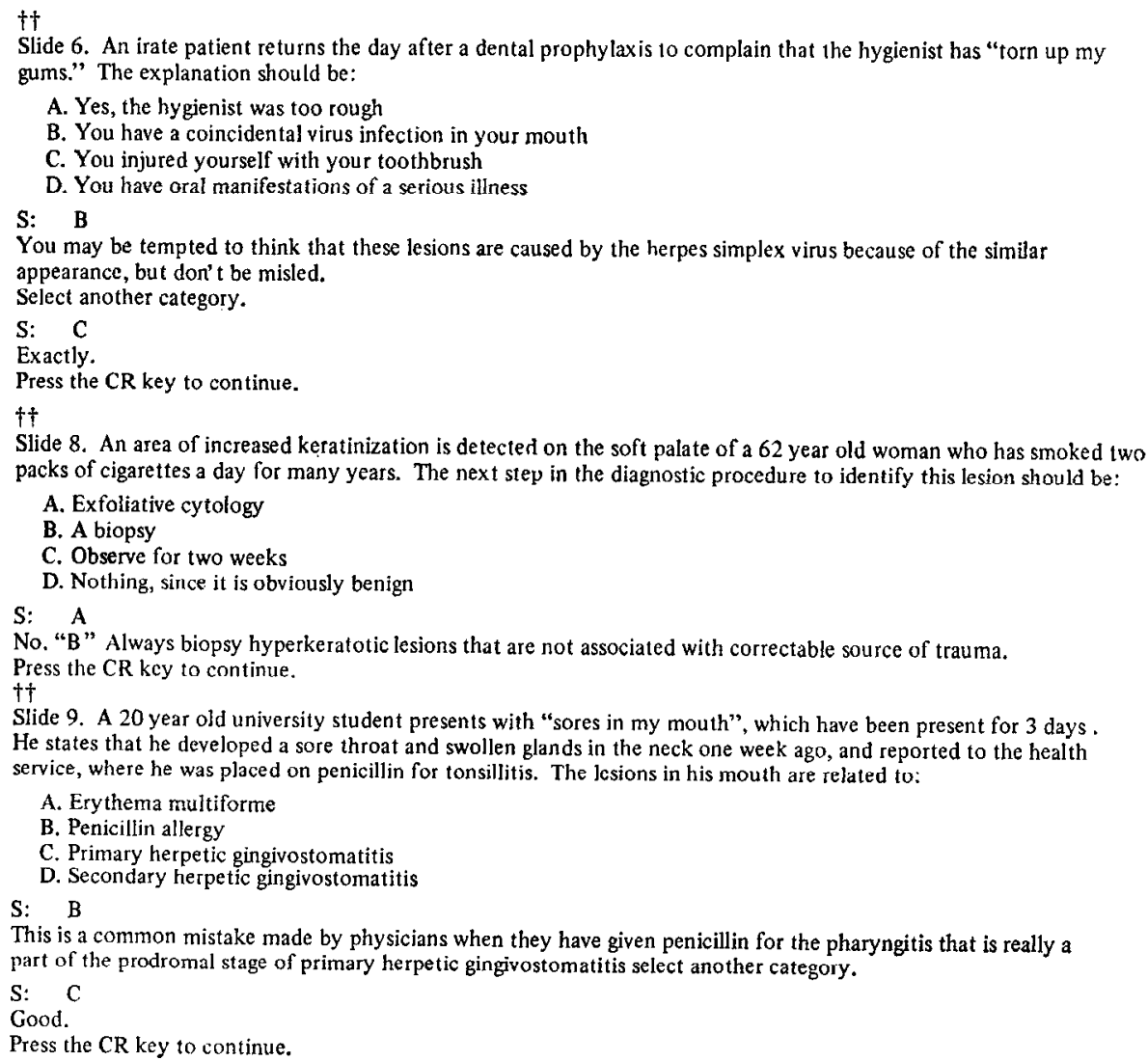


of patients they can see during their rotation in Diagnosis Clinic and even then their experience is limited by the mix of patients who gain admission to the clinic while that student is there. Dental students are taught a great deal of oral medicine and will be required to make fine discriminations among a wide variety of problems in practice.

One solution. It is possible but difficult to call all dental students into the Oral Diagnosis Clinic when an unusual disorder is found. If each student were asked to offer a diagnosis to

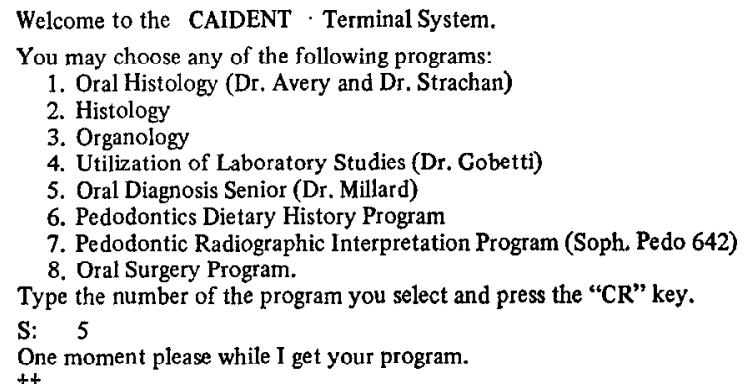
answer some questions about. Just type in your answers, and press the CR key at the end of each answer. Have you used this program before?

S: No

$+1$

Let's start with Slide 1.

This 56 year old caucasian male presented with a complaint of a "sore on the right side of my tongue" of three weeks duration. On examination, the area of ulceration is firm and somewhat tender to palpation. The next step in determining the nature of the tissue change is:
A. A biopsy
B. Observe for 10 days for evidence of healing, then bio sy if indicated.
C. Serologic test for syphilis.
D. Exfoliative cytology.

\section{S: A}

The clinical features of this lesion make it difficult to distinguish it from a carcinoma. However, its short duration suggests traumatic origin. Because of the probable source and because cells can be obtained for cxamination by exfoliative cytology technic, a cytology specimen is the preferred step at this point. Press the CR key to continue.

$\dagger \dagger$

Slide 2. A 21 year old white male complained of the first episode of a sore mouth which appeared abruptly two weeks ago. He denied the presence of lesions elsewhere on his body, but stated that his eyes "mattered" and were "stuck closed" when he woke in the morning. He has no fever and the complete blood count is normal in all respects. The diagnosis is most likely:

A. Stomatitis venenata.

B. Primary herpetic gingivostomatitis

C. Pemphigus

D. Erythema multiforme

S: D

Good. Press the CR key to continue

tt

Slide 3. A 41 year old biack female presented for routine dental examination. Her radiographs reveal significant changes in the mandibular incisor region. The area is asymptomatic and clear clinically. In order to establish a diagnosis, the dentist should do:

A. A biopsy.

B. Pulpal vitality tests.

C. An opening into the pulp chamber.

D. Percussion.

Sine. Press the CR key to continue.

${ }^{1}$ This program was developed by Dr. H. Dean Millard, Chairman, Department of Oral Diagnosis and Radiology, School of Dentistry, University of Michigan. This material is reprinted here with his permission. The material was programmed by Judson Spencer. 
an instructor and then could discuss the alternatives for a few minutes they might then have very broad exercise of their abilities but the logistic problems of interrupting students performing elsewhere and the faculty time required would be prohibitive.

Another possibility includes photographing all unusual cases and making these files available to students, but there is still the problem of explaining why a given choice is incorrect. In many cases, students learn a great deal from an explanation of why their choice was incorrect.

The CAI solution. Students are presented with a slide which illustrates the salient visual information of a case and they are given a brief description of the case. Based on this information they are asked to identify the best diagnosis or select a strategy that will provide more information. An example of this interaction appears below.

Discussion. The advantages of using a computer to assist in teaching/testing this skill include:

(1) the adaptability of the program to the student's response. If the student is correct he may continue immediately to the next set; if incorrect, the reason is explained and he is usually asked to then enter the correct response.

(2) Time. Students may and almost always do require varying amounts of time to arrive at a diagnosis. If this activity is conducted in a group setting some students will be bored with repetition of what they already know and others will prefer to explore the reasons for ruling out other plausible diagnoses. As a result, few students will be using their time effectively.

(3) A diagnosis can be made on the basis of salient data only. To replicate these experiences in the clinic would require substantial "overhead" procedures of calling a patient from the waiting room, seating the patient, scrubbing up and other time-consuming tasks with which the student is already intimately familiar.

(4) Repetition. The object of this experience is to present all students with situations that cover as many diagnostic problems as possible. To do this, all students must have the opportunity to be presented with these problems and that requires repetition which does not need an instructor; it can be handled very effectively by a computer.

\section{DISCUSSION}

These brief examples illustrate some of the salient features of CAI in Dentistry. The practice of Dentistry is a complex mix of psychomotor, cognitive and affective skills. Students receive much individual obscrvation and corrective fccdback during their psychomotor learning experiences, and come to their dental curriculum with practice in the study skills needed for much of the didactic coursework. What is new to a large extent is their need to synthesize information from a number of sources to determine a plan of action. It is this experience of synthesis in which individual attention is needed and which CAI can provide.

A few years ago Stanford University sponsored a CAI project in a school that served a low-income community. While this author was visiting the project, he had the opportunity to ask an 8-yr-old how he felt about learning from a computer. The answer was: I like it because the computer doesn't care if I smell; my teacher does." This comment inspired further thought about the characteristics of the learner in the educational environment. Many students are hesitant to ask a "dumb" question or to confirm their suspicion that certain information is not necessary to a decision. CAI provides a non-threatening, non- 
evaluative environment in which students can explore alternatives without concern for criticism or "image". In many cases students need to know what is not important so that they can better focus on and remember what is.

$I$ predict that CAI will be used increasingly in health science education for a number of reasons. The content lends itself to the decision-making, branching format of CAI very well; the need for health science manpower is currently exceeding the ability to supply these personnel; the "information explosion" is upon us and, to a certain extent, we have not accommodated its demands; and computer technology now indicates that terminals and computer access will soon be within the economic and practical grasp of a vast variety of professionals for a multitude of uses. When that time comes, computers will be used for briefings on new developments in health science, information retrieval, corroborative diagnoses, coordination of emergencies, and continuing education. A recent survey by BrIGHAM, KAMP and Cross ${ }^{(1)}$ indicates that 48 of 52 dental schools responded; 11 reported using CAI and 12 indicated that they would be using it in the future. It seems to me that the 29 schools remaining might want to ask themselves why they haven't investigated this medium. It's not to jump on the bandwagon; it's because there are distinct advantages and uses of CAI.

\section{SUMMARY}

The CAIDENT Project at the School of Dentistry of the University of Michigan began as a result of early developmental work in computer-assisted instruction (CAI) and the commitment to establishing a flexible, modular curriculum for self-paced learning. Of more than 4000 clock hours in the current dental curriculum, 760 (19 per cent) have been identified as amenable to delivery by individualized instruction in general, and $258 \mathrm{hr}$ ( 6 per cent of the total curriculum) have been identified for CAI delivery. CAI is one of several media in the project, including videotape, slide/tape, audio-tutorial, and programmed instruction.

The project was funded by the Bureau of Health Manpower on 8 Augusl 1972 and is designed to operate for $5 \mathrm{yr}$. The grant for the project provides for 41 persons, 32 computer terminals and a completely equipped learning laboratory. Faculty are encouraged to participate in the preparation of materials in a variety of ways which are listed and explained.

Dentistry is both an art and a science, and very high standards of performance are required and expected. It is proposed that individualized instruction in general and CAI in particular will provide an opportunity for the optimalization of learning for all students. The uses of CAI in dentistry are most appropriate where: (1) students require substantially different amount of time to learn; (2) different responses are made by students to the same question; (3) students need to explore the effects of various decision strategies; (4) actions taken on the basis of student response can be identified; (5) each student is required to actively participate in the specification of or commitment to a particular decision; (6) it is cumbersome, difficult or unnecessary to present all cues which comprise a particular problem situation; and (7) material is substantially repetitive.

Two examples of student-computer interaction are given which illustrate the mechanism of allowing a student to express an opinion or make a judgment where the computer responds with reasons why the choice was correct or incorrect.

A prediction is made of the increased use of computers in health science education. 


\section{REFERENCE}

1. Christopher R. Brigham, Martin Kamp and Richard J. Cross, A Guide to Computer-Assisted Instruction in the Health Sciences, p. 325. National Technical Information Service, Springfield, Virginia (December 1972). 